\title{
Ghrelin mediates stress-induced food-reward behavior in mice
}

\author{
Jen-Chieh Chuang, ${ }^{1}$ Mario Perello, ${ }^{1}$ Ichiro Sakata, ${ }^{1}$ Sherri Osborne-Lawrence, ${ }^{1}$ \\ Joseph M. Savitt, ${ }^{2}$ Michael Lutter, ${ }^{1,3}$ and Jeffrey M. Zigman',3,4
}

\begin{abstract}
1Division of Hypothalamic Research, Department of Internal Medicine, The University of Texas Southwestern Medical Center, Dallas, Texas, USA.
2Department of Neurology, The Johns Hopkins University School of Medicine, Baltimore, Maryland, USA. ${ }^{3}$ Department of Psychiatry, The University of Texas Southwestern Medical Center, Dallas, Texas, USA. ${ }^{4}$ Divsion of Endocrinology and Metabolism, Department of Internal Medicine, The University of Texas Southwestern Medical Center, Dallas, Texas, USA.
\end{abstract}

\begin{abstract}
The popular media and personal anecdotes are rich with examples of stress-induced eating of calorically dense "comfort foods." Such behavioral reactions likely contribute to the increased prevalence of obesity in humans experiencing chronic stress or atypical depression. However, the molecular substrates and neurocircuits controlling the complex behaviors responsible for stress-based eating remain mostly unknown, and few animal models have been described for probing the mechanisms orchestrating this response. Here, we describe a system in which food-reward behavior, assessed using a conditioned place preference (CPP) task, is monitored in mice after exposure to chronic social defeat stress (CSDS), a model of prolonged psychosocial stress, featuring aspects of major depression and posttraumatic stress disorder. Under this regime, CSDS increased both CPP for and intake of high-fat diet, and stress-induced food-reward behavior was dependent on signaling by the peptide hormone ghrelin. Also, signaling specifically in catecholaminergic neurons mediated not only ghrelin's orexigenic, antidepressant-like, and food-reward behavioral effects, but also was sufficient to mediate stress-induced food-reward behavior. Thus, this mouse model has allowed us to ascribe a role for ghrelin-engaged catecholaminergic neurons in stress-induced eating.
\end{abstract}

\section{Introduction}

Most humans experience altered feeding behaviors upon stress, with approximately $40 \%$ eating more and $40 \%$ eating less than usual (1). Furthermore, upon stress, most people report an increase in the intake of highly palatable foods, independent of hyperphagia or hypophagia $(2,3)$. Altered eating is also a frequent finding in individuals with major depressive disorder, with the "atypical" subtype even containing hyperphagia as a possible distinguishing characteristic (4). In one study, $46 \%$ of study subjects who met DSM-IV criteria for major depressive disorder with atypical features reported increased appetite (5). Of the remaining depressed patients without atypical features, $18 \%$ reported increased appetite, while $50 \%$ reported decreased appetite (5). The complex eating behaviors that are associated with and/or stimulated by stress and major depression undoubtedly contribute to the increased number of overweight and obese individuals who experience or have experienced stress and depression. For example, a longitudinal study from New Zealand showed that major depression in late-adolescent girls was associated with a 2.3-fold increased risk of obesity in adulthood and, furthermore, that the prevalence of obesity in adulthood was positively correlated with the number of major depressive episodes during adolescence in these girls (6). In another study, $47 \%$ of a large cohort of subjects with atypical depression reported increased body weight (5). Also, the combined overweight and obesity prevalence in a sample of US veterans with posttraumatic stress disorder was found in a chart review study to exceed that within the US general population by $20 \%$ (7).

Authorship note: Jen-Chieh Chuang and Mario Perello contributed equally to this work.

Conflict of interest: The authors have declared that no conflict of interest exists. Citation for this article: J Clin Invest. 2011;121(7):2684-2692. doi:10.1172/JCI57660.
Relatively few animal models have been developed to adequately explore the mechanisms responsible for complex, stress-induced and depression-associated eating behaviors. Animal models to investigate stress-based eating have included repeated bouts of restraint in rats, which was shown to increase intake of a freely available lard- and sucrose-based chow (8), and also a visible burrow system in rats, which led to increased meal size in subordinate males (9). A third model involved measuring intake of high-fat diet (HFD) pellets to which mice exposed to chronic variable stress were given limited access along with preweighed regular chow $(10,11)$. In this latter model, mice with genetic deletion of corticotrophin-releasing factor receptor-2, which results in an exaggerated hypothalamic-pituitary-adrenal response to stress, showed an increase in HFD consumption during chronic variable stress (11). Furthermore, wild-type mice that previously had been calorically restricted and then were allowed to recover body weight consumed a significantly greater proportion of high-fat calories during chronic variable stress than did wild-type mice without previous calorie restriction (10). While these models have provided important insights into stress-induced changes in feeding, no study has yet incorporated strategies to examine the more complex, motivation- and learning-associated decision-making processes likely at work in humans subjected to stress.

The lack of appropriate animal models, and, in particular (until recently; refs. 10,11), mouse models, has also limited identification of the actual molecular substrates involved in these stressinduced eating behaviors. Thus, these substrates remain mostly undetermined. Emerging literature does suggest an association between stress and signaling by ghrelin, a peptide hormone produced primarily by distinct ghrelin cells located in the stomach and elsewhere in the gastrointestinal tract (12-15). Ghrelin's roles in promoting food intake and positive energy balance in response 
to caloric restriction have been documented in numerous studies (16-18). The mechanisms by which ghrelin promotes food intake are multifaceted and include enhancing the rewarding properties of certain foods (19-24). For instance, conditioned place preference (CPP) for HFD, in which an animal will gravitate toward a chamber with environmental cues it has associated with a HFD reward, and operant nose poking for HFD, in which a motivated animal will exert increased effort (by poking a button with its nose) to receive a HFD reward, are induced in mice by peripheral ghrelin injection and prolonged caloric restriction, both of which increase plasma ghrelin $(21,23)$. Similarly, i.p. or i.c.v. ghrelin administration increases operant lever pressing for sucrose pellets in rats (24). Ghrelin receptor (growth hormone secretagogue receptor [GHSR]) antagonist administration and genetic deletion of GHSRs, though, prevents caloric restriction-associated CPP for HFD in mice (21, 23). GHSR antagonist administration also prevents caloric restriction-associated operant lever pressing for sucrose in rats (24). These findings suggest an obligatory role for ghrelin in caloric restrictionassociated food-reward behaviors. Supporting these findings, ghrelin injection preferentially enhances fat intake over carbohydrate intake in rats (22). Ghrelin administration increases consumption of palatable saccharin solution and increases preference for saccharin-flavored foods in wild-type mice but not GHSR-deficient mice (19). GHSR-deficient mice and GHSR antagonist-treated rats consume less peanut butter or Ensure but do not decrease consumption of regular chow in a free choice protocol (20). GHSR antagonist blocks CPP for chocolate pellets in satiated rats (20). Also, ghrelin administration to human subjects during functional magnetic resonance imaging increases the neural response to food pictures in brain regions implicated in hedonic feeding, confirming its importance in human appetite regulation (25).

Regarding a potential role for ghrelin in stress-based eating, elevations of ghrelin have been observed in several animal and human stress models (26-31). For instance, human subjects subjected to the standardized trier social stress test display increased plasma ghrelin (30). The chronic social defeat stress (CSDS) procedure, which subjects male mice to repeated bouts of social subordination by an older and larger aggressor male, also leads to sustained plasma ghrelin elevations $(26,32,33)$. After CSDS, most C57BL/6J male mice exhibit lasting depression-like behavioral deficits, including social withdrawal (as measured using a social interaction task), which can be reversed by chronic, but not acute, antidepressant treatment (32). CSDS is thus considered a model of prolonged psychosocial stress in humans, featuring aspects of major depression and posttraumatic stress disorder $(32,33)$. Of note, genetic deletion of GHSRs exacerbates depression-like behaviors normally induced by CSDS, while, conversely, pharmacologic and certain physiologic (via chronic caloric restriction) ghrelin elevations produce antidepressant-like behavioral effects (26). Furthermore, CSDS is associated with hyperphagia by mice with access to freely available regular chow both during (26) and for at least 1 month after (26, $34,35)$ the defeat period. This hyperphagia, which is not observed in mice lacking GHSRs, may contribute to the higher body weight gain observed in CSDS-exposed C57BL/6J mice $(26,34,35)$.

Here, we describe what we believe to be a new model, in which food-reward behavior, as assessed using a CPP task, is measured in mice after exposure to CSDS. We also have assessed the relationship of stress-induced food-reward behavior to elevations in circulating levels of the peptide hormone ghrelin, because of the aforementioned studies demonstrating rises in ghrelin after various forms of stress, including CSDS $(26,31)$, and ghrelin-enhanced preference for, place preference for, and operant responding for pleasurable, energy-dense foods (19-22). We demonstrate that CSDS in mice increases both CPP for and intake of HFD. We also demonstrate that such stress-induced food-reward behavior is dependent on ghrelin signaling, as it is not observed in mice lacking ghrelin receptors (GHSRs). Finally, we demonstrate that ghrelin signaling specifically in catecholaminergic neurons not only mediates certain of ghrelin's orexigenic, antidepressant-like, and food-reward behavioral effects, but also is sufficient to mediate stress-induced food-reward behavior.

\section{Results}

Development of a mouse model of stress-induced complex eating behaviors and determination of the role of ghrelin in these behaviors. Based upon the observations that circulating ghrelin levels rise after various forms of stress, including CSDS $(26,31)$, and that ghrelin signaling increases the rewarding properties of energy-dense foods (19-22), we hypothesized that stress-induced elevations in ghrelin mediate reward behaviors aimed at obtaining calorically dense, pleasurable comfort foods upon social stress. To test this hypothesis, we developed what we believe to be a new mouse model to probe the molecular substrates and neurocircuits mediating stress-induced food-reward behavior. This model involved assessing CPP for HFD by mice after their exposure to CSDS.

We began by using both wild-type and ghrelin receptor-deficient (GHSR-null) littermates. As mentioned, CSDS results in several persisting behavioral deficits reminiscent of depression, including social isolation (Figure 1, A and B, and refs. 32, 33). Consistent with previous findings (26), CSDS-induced social isolation was intensified by genetic deletion of GHSRs (Figure 1, A and B). CSDS resulted in elevations of acyl-ghrelin (the confirmed ligand for GHSR), when assessed 1 day after the social interaction test (as had been reported previously; ref. 26), but not desacyl-ghrelin (Figure 1, C and D). Similar effects on ghrelin levels were observed in GHSR-null mice (Figure 1, C and D), suggesting that lack of ghrelin signaling does not impact plasma ghrelin in this physiologic setting. Also, corticosterone levels measured 1 day after the social interaction test were higher in wild-type mice than in those in GHSR-null littermates after CSDS (Figure 1E). This seems relevant to the differences in stress-associated, reward-based eating observed in wild-type versus GHSR-null littermates (described below), because glucocorticoid secretion intensifies motivated behaviors and increases intake of highly palatable foods (3).

One day after measurement of social interaction, CSDS- and non-CSDS-exposed mice were placed into a CPP for HFD protocol (21). After CSDS, wild-type mice demonstrated an increased preference for the chamber previously paired with HFD (Figure 2A), as had been previously demonstrated in wild-type mice exposed to other manipulations that raise ghrelin, including calorie restriction and ghrelin administration (21). In contrast, neither CSDSexposed GHSR-null mice nor non-CSDS-exposed control subjects demonstrated CPP for HFD (Figure 2A).

Furthermore, CSDS-exposed wild-type mice gained more weight over the course of the CPP protocol than did control mice (as had been shown previously; refs. 34, 35) or GHSR-null mice (Figure 2B). Here, this observed increased body weight partly may be contributed to by a selective increased intake of HFD during the CPP conditioning sessions: the CSDS-exposed wild-type mice consumed more HFD during the conditioning sessions than did 

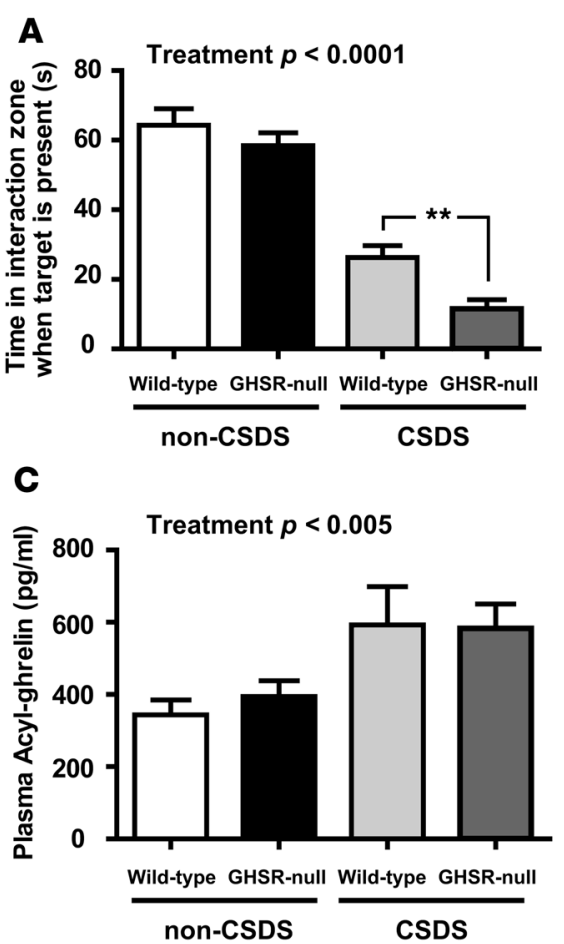

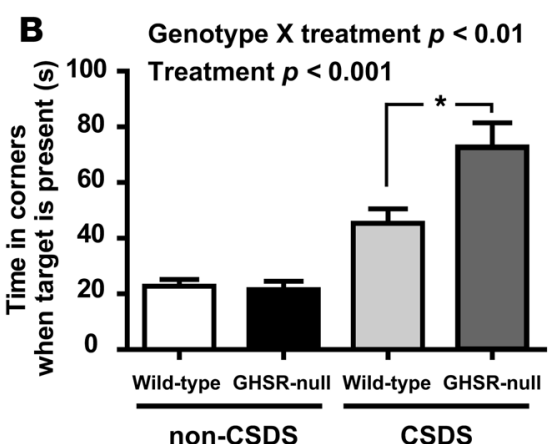

D

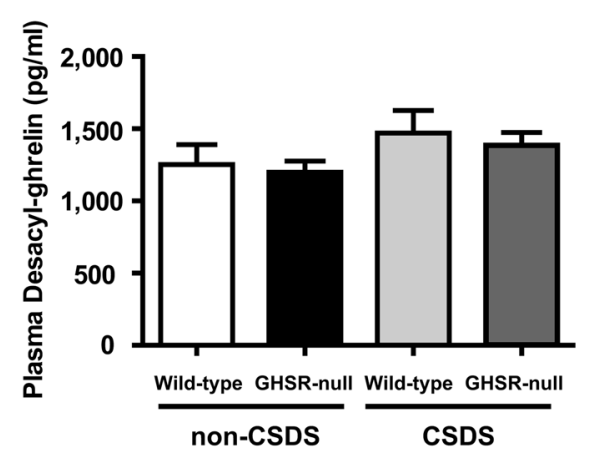

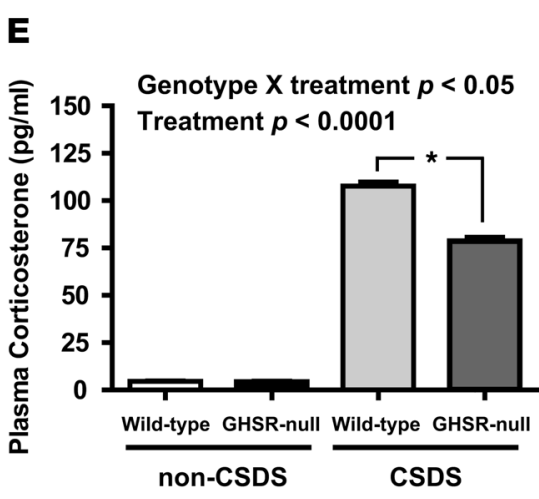

Figure 1

Intact acyl-ghrelin signaling via GHSRs minimizes CSDS-associated, depression-like behavior. CSDS induces depression-like behaviors, as indicated by $(\mathbf{A})$ decreased time subjects spend in the interaction zone near the target mouse and (B) increased time subjects spend in the corners of the social interaction test arena, as compared with that of non-CSDS-exposed mice ( $n=20$ per group). GHSR deletion aggravates these behaviors $\left({ }^{\star} P<0.05,{ }^{*} P=0.0012\right)$. (C) CSDS increases plasma acyl-ghrelin (D) but not desacyl-ghrelin, in wild-type and GHSR-null mice ( $n=6-7$ per group). (E) CSDS increases plasma corticosterone, but this effect is not as pronounced in GHSR-null mice ( ${ }^{\star} P<0.05 ; n=15-18$ per group). Significant effects of treatment (CSDS vs. control non-CSDS) and significant genotype $X$ treatment interactions are indicated.

the other groups (Figure 2C). Consistent with other experiments, demonstrating a selective effect of GHSR deletion on peanut butter consumption but no effect on regular chow consumption in mice offered a free choice between those 2 diets (20), no differences were observed here in consumption of regular chow available during the conditioning sessions (Figure 2D).

Importantly, previous control experiments (21), in which we assessed ghrelin's pharmacological ability to induce CPP for HFD, determined that similar CPP results could be obtained upon reversal of order of exposure to HFD and regular chow (in other words, conditioning with HFD on even days instead of on odd days) or upon use of quantities of HFD and regular chow that matched the mean amount of regular chow eaten during the conditioning sessions (instead of using equal calorie amounts of HFD and regular chow, as was done here). This suggests that it is the type of pellet rather than the order of pellet exposure or amount of food eaten during the conditioning sessions that determines the place preference in the task (21).

Determination of the role of ghrelin receptor-expressing catecholaminergic neurons in ghrelin action. We next sought to determine neuronal subtypes and brain regions mediating ghrelin's actions on stressinduced food reward. GHSRs have a well-defined central expression pattern, which includes tyrosine hydroxylase-expressing (THexpressing) ventral tegmental area (VTA) neurons $(36,37)$. Extensive literature attributes essential roles to the VTA and its dopaminergic projections in mediating both reward behavior of various types and mood regulation, including that influenced by $\operatorname{CSDS}(38,39)$. Several studies also have demonstrated that ghrelin can act on these VTA neurons to influence food intake and food reward (20, 36, 40-44).

Here, we assessed the role of direct action of ghrelin on VTA and other catecholaminergic neurons by generating mice in which GHSRs are expressed selectively at those sites. This was achieved by crossing GHSR-null mice, which contain a loxP-flanked transcriptional blocking cassette inserted into the Ghsr gene, to mice in which Cre recombinase expression is driven by the $\mathrm{TH}$ promoter (TH-Cre mice) $(45,46)$. Study mice derived from this genetic cross, including wild-type mice (which contain GHSRs in all usual GHSR expression sites, with or without the TH-Cre transgene), GHSRnull mice (which lack GHSRs), and GHSR-null/TH mice (which express endogenous levels of GHSRs only in TH-containing neurons programmed to express both GHSR and $\mathrm{TH}$ ), were previously used to demonstrate ghrelin-mediated protection against substantia nigra cell loss and striatal dopamine loss after administration of the neurotoxin MPTP (47). Predicted patterns of TH cell-restricted GHSR expression within GHSR-null/TH mice were confirmed by real-time quantitative reverse transcription PCR (qRT-PCR) on tissue punches from different brain regions and by in situ hybridization histochemistry on coronal brain sections. In particular, within GHSR-null/TH mice, GHSR expression was observed in the VTA and arcuate nucleus (ARC) but not in sites known to lack $\mathrm{TH}$-expressing neurons and/or GHSR-expressing neurons within wild-type mice, such as the dentate gyrus (DG), nucleus accumbens 
A


B

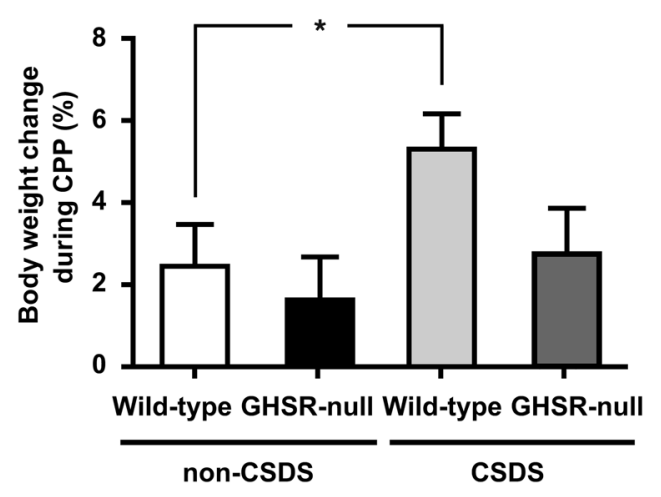

D

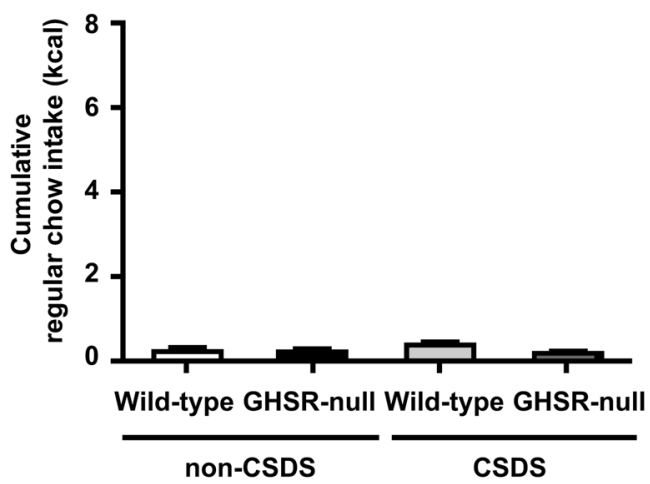

Figure 2

CSDS enhances the rewarding properties of HFD, consumption of HFD reward pellets, and body weight gain in a ghrelin-dependent manner. (A) Unlike non-CSDS-exposed, control wild-type mice, CSDS-exposed wild-type mice demonstrate CPP for HFD $\left({ }^{\star \star} P=0.021\right)$. CSDS-induced HFD reward behavior is not observed in GHSR-null littermates ( $n=20$ per group). (B) CSDS-exposed wild-type mice gain more weight during the conditioning period than do the other groups $\left({ }^{\star} P=0.039\right)(n=12$ per group). (C) CSDS-exposed wild-type mice consume more HFD during CPP conditioning sessions than do non-CSDS-exposed control mice ( $\left.{ }^{* *} P=0.006\right)$; such enhanced HFD intake is not observed in CSDS-exposed GHSR-null mice ( $n=12$ per group). (D) Consumption of the regular chow pellets available during CPP conditioning sessions is unaffected by CSDS in either genotype ( $n=12$ per group).

(NAC), or suprachiasmatic nucleus (SCN) (Figure 3A and Supplemental Figure 1; supplemental material available online with this article; doi:10.1172/JCI57660DS1). Of note, GHSR expression levels within VTA and ARC tissue punches were not as high as those in the corresponding wild-type tissues, since GHSR expression within wild-type VTA and ARC also occurs in other, non-TH cell types (36, $44,48)$. Other sites of presumed usual GHSR-TH coexpression, in which TH-Cre-driven GHSR expression was observed by in situ hybridization histochemistry within GHSR-null/TH mice, included the substantia nigra, a few other hypothalamic sites (anteroventral periventricular nucleus, dorsomedial nucleus, and capsule of the ventromedial nucleus), and scattered caudal brainstem (nucleus of the solitary tract) cells, as reported previously (data not shown; ref. 47). The TH-containing neurons in all of these sites are dopaminergic, with the exception of the nucleus of the solitary tract TH-neurons, which, based on studies in the rat, could be either adrenergic, noradrenergic, or dopaminergic (49).

As demonstrated previously $(26,45)$, wild-type mice, but not GHSR-null mice, responded to s.c. injections of ghrelin by increasing their intake of freely available regular chow (Figure 3B). Reexpression of GHSR in TH-containing neurons partially restored ghrelin-induced food intake (Figure 3B). Similar to previous work $(45,50)$, fasting blood glucose levels were lower in GHSR-null mice than in wild-type littermates (Figure 3C). However, GHSR-null/ TH mice showed similar fasting blood glucose levels to those of GHSR-null mice, suggesting that direct action by ghrelin on catecholaminergic neurons does not mediate its modulatory effects on glycemia (Figure 3C). Selective catecholaminergic GHSR expression also was shown to rescue ghrelin's pharmacological ability to induce CPP for HFD (Figure 3D).

We next analyzed the effects of CSDS on social interaction and food intake by GHSR-null/TH mice. Consistent with previous findings, GHSR-null mice showed increased social isolation after CSDS compared with that of wild-type littermates (Figure 3E). Reexpression of GHSR in TH-containing neurons improved social interaction scores to levels similar to those observed in wild-type mice (Figure 3E), suggesting that catecholaminergic GHSR expression is sufficient to mediate ghrelin's antidepressant actions. Finally, we assessed the performance of a separate cohort of mice in the CPP for HFD protocol after CSDS. Selective catecholaminergic GHSR expression was sufficient to restore the chronic stress induction of CPP for HFD (Figure 3F). 
A
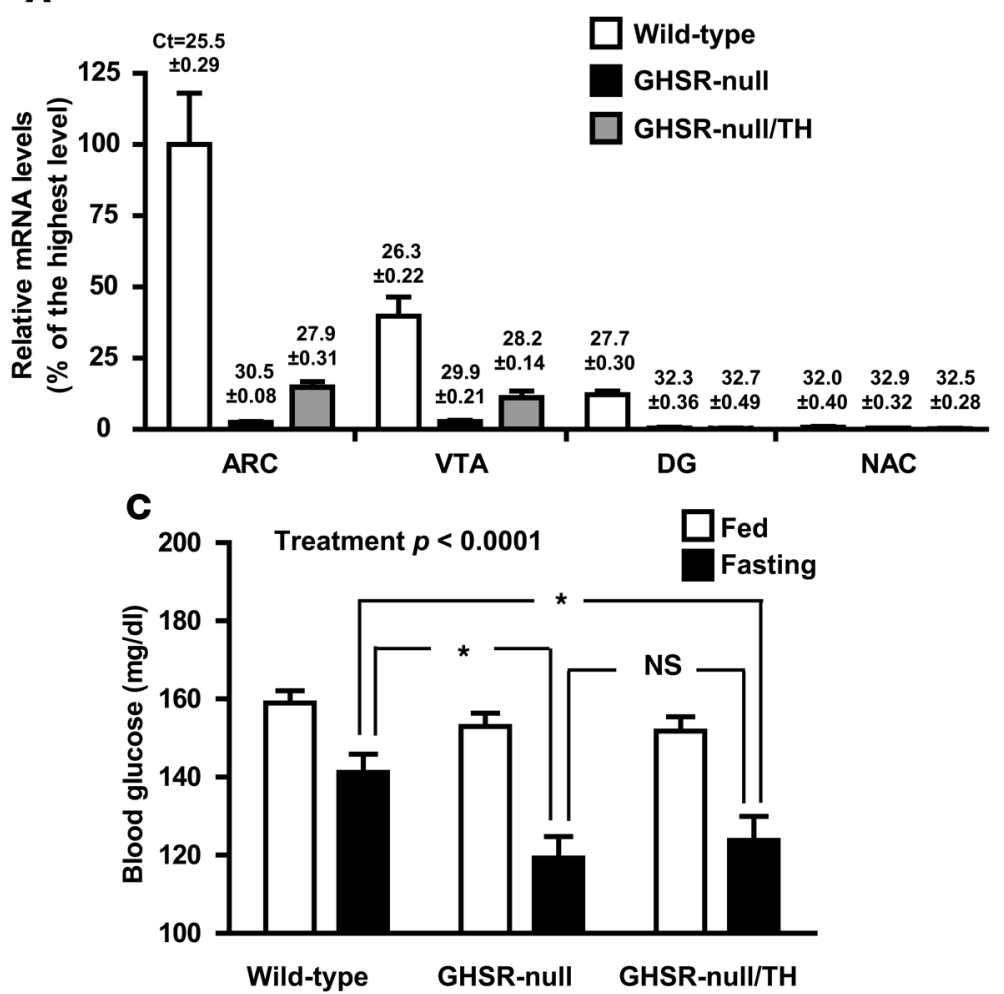

$\mathbf{E}$

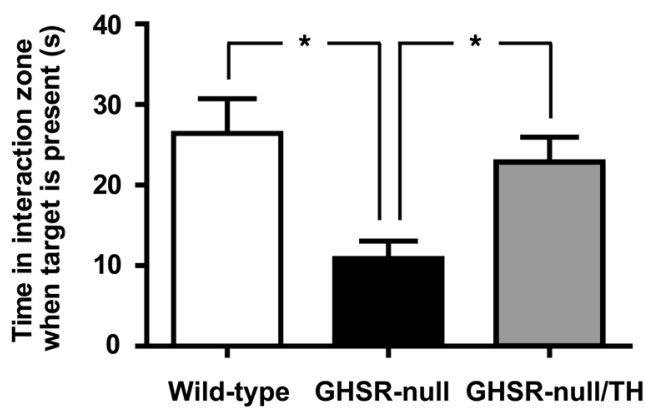

B Genotype X treatment $p<0.0001$

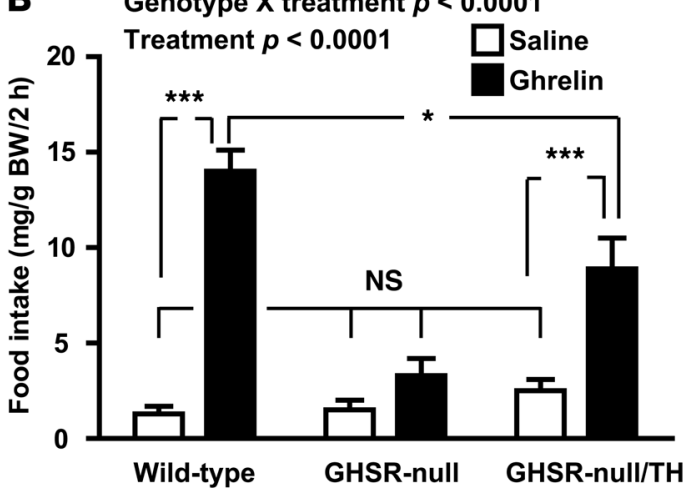

D

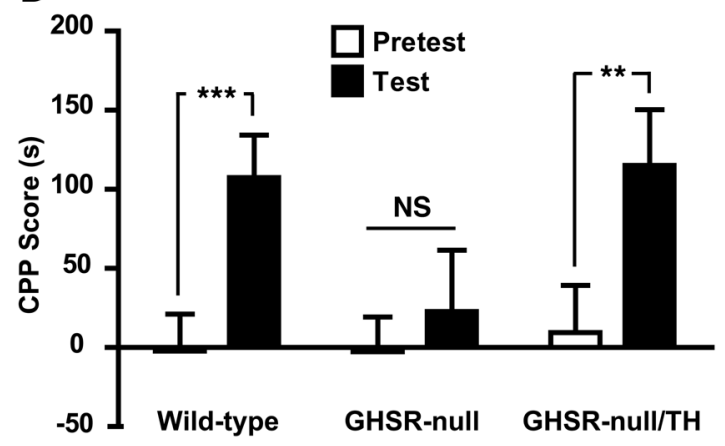

$\mathbf{F}$

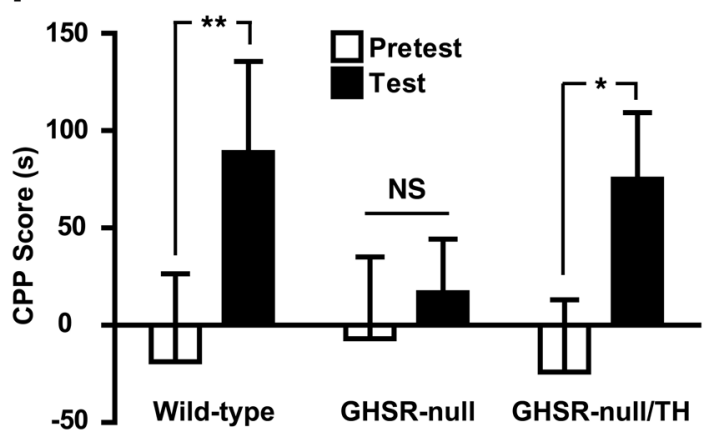

Figure 3

Selective GHSR expression in catecholaminergic neurons is sufficient for several ghrelin-mediated actions. (A) Cre-mediated restoration of Ghsr mRNA expression is observed in brain regions of GHSR-null/TH mice containing catecholaminergic neurons programmed to express GHSRs, including the ARC and VTA, as determined using qRT-PCR ( $n=6$ per group). Mean \pm SEM threshold cycle values for Ghsr mRNA in each brain site are denoted above the bars. (B) Ghrelin-induced acute food intake is partially restored in GHSR-null/TH mice. ( ${ }^{\star} P<0.05,{ }^{* * *} P<0.0001$ ) (C) Fasting blood glucose levels are not restored to wild-type levels in GHSR-null/TH mice $\left({ }^{\star} P<0.05\right)$. (D) Administered ghrelin-induced CPP for HFD $\left({ }^{* *} P<0.005,{ }^{* *} P<0.0001\right)$, (E) social interaction phenotype after CSDS $\left({ }^{\star} P<0.05\right)$, and (F) CSDS-induced CPP for HFD $\left({ }^{\star} P=0.042\right.$, ${ }^{\star \star} P=0.020$ ) are all restored to wild-type levels in GHSR-null/TH mice. For $\mathbf{B}-\mathbf{F}, n=19-30$ wild-type mice (including 12-22 mice without and 5-14 mice with TH-cre), $n=14-34$ GHSR-null mice, and $n=14-23$ GHSR-null/TH mice. Significant effects of treatment (ghrelin vs. saline or ad libitum-fed versus 24-hour fast) and significant genotype $X$ treatment interactions are indicated.

\section{Discussion}

In summary, to our knowledge, the CSDS-induced CPP for HFD protocol described here is the first reported mouse model of an antidepressant-responsive, neuropsychiatric syndrome used to probe the mechanisms responsible for hedonic eating behaviors that occur upon chronic stress $(32,33)$. As mentioned, the CSDS protocol is considered a fairly representative model of prolonged psychosocial stress in humans and also produces a depression-like state, which, similar to major depressive disorder in humans, is known to be reversible only upon chronic, but not acute, administration of antidepressant agents $(32,33)$. Here, using a CPP protocol to examine complex, reward eating behaviors induced by the chronic stress/depression model, we were able to demonstrate stress-associated increases in both CPP for and intake of HFD. Thus, exposure of mice to CSDS prior to the CPP for HFD protocol models the complex changes in eating behavior and body weight gain associated with chronic stress and major depressive disorder - particularly the atypical subtype - in humans $(1,2,5,7)$. 


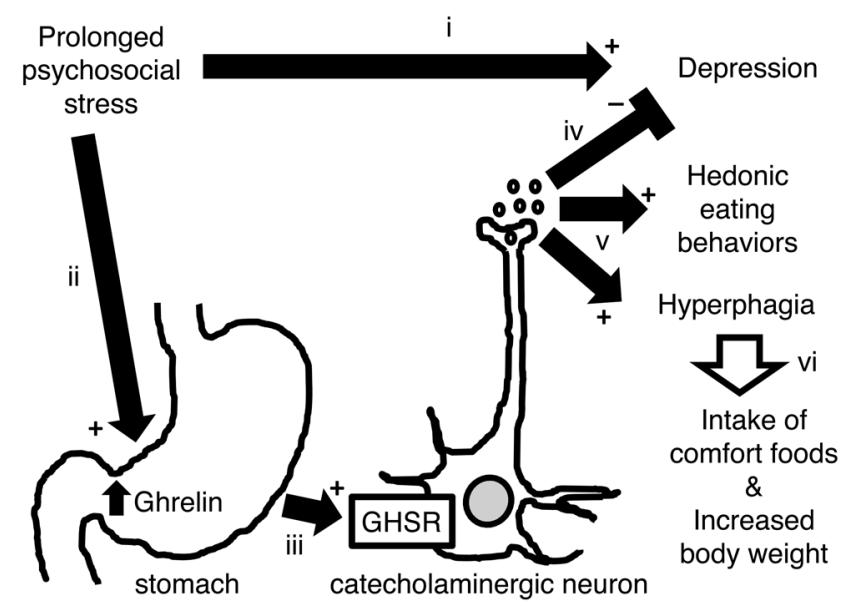

Figure 4

Model of ghrelin's roles in stress-induced behaviors. (i) CSDS in mice, which is a model of prolonged psychosocial stress in humans, results in several persisting behavioral deficits reminiscent of depression. (ii) Psychosocial stress also leads to elevations in circulating levels of ghrelin, which, in turn (iii), interacts with GHSRs localized to catecholaminergic neurons in the brain (the exact expression site[s] of these GHSR-containing, catecholaminergic neurons - most of which are dopaminergic - has not been identified but likely include the VTA). (iv) Engagement of these neurons by ghrelin induces a series of changes that minimizes what would otherwise be worsened depression-like behaviors, while at the same time, ( $v$ ) induces HFD food-reward behavior and hyperphagia, leading to (vi) increased intake of highly palatable, calorically dense comfort foods and increased body weight.

Also, this model revealed a dependence of these food-reward behaviors on intact ghrelin signaling via GHSRs.

In addition, to our knowledge, this study contains the first major use of mice with conditionally manipulated ghrelin receptor expression. More specifically, we showed that direct ghrelin signaling via GHSRs localized to catecholaminergic neurons was sufficient not only for CPP for HFD induced by ghrelin administration and for usual mood responses after chronic stress but also for stress-induced food-reward behaviors.

A schematic model, which incorporates these findings with some previous results and which describes the proposed role of ghrelin and GHSR-TH-coexpressing neurons in stress-induced depression and eating behaviors, appears in Figure 4 and is further described as follows. We, and others, have shown that the CSDS protocol in mice, which is a model of prolonged psychosocial stress in humans, results in several persisting behavioral deficits reminiscent of depression (refs. 26, 32, 33, and Figure 4, part i). Psychosocial stress also led to elevations in circulating levels of acyl-ghrelin, via a pathway which has not yet been elucidated (Figure 4, part ii). (Speculatively, this stress-induced elevation may involve stimulation of $\beta_{1}$-adrenergic receptors on ghrelin cells, as such a pathway has been shown to play a role in ghrelin release during a 24-hour fast and as increased sympathoadrenal tone is a known consequence of stress [51-53]). Ghrelin, in turn, interacts with its receptor, GHSR, which is found distributed throughout the brain and periphery in a specific pattern (37). The data in this report suggest that direct ghrelin interaction, specifically with GHSR-TH-coexpressing neurons (Figure 4, part iii), is sufficient for many of the behaviors induced in the setting of prolonged psychosocial stress.
In particular, engagement of these catecholaminergic neurons by ghrelin minimized what would otherwise be worsened depression (as evidenced by the increased social avoidance in mice lacking GHSRs after CSDS; Figure 4, part iv), while at the same time, induced various hedonic eating behaviors and hyperphagia (Figure 4, part v). Of note, direct ghrelin action on catecholaminergic neurons did not appear to mediate ghrelin's effects on glycemia, as fasting blood glucose levels were similarly and significantly lower than wild-type levels in both GHSR-null and GHSR-null/TH mice. The effects on eating behavior led to increased intake of highly palatable, calorically dense foods, which in the human experience are often termed comfort foods, and increased body weight (Figure 4, parti vi), which in turn can result in overweight and obesity.

Although the current study does focus in on the identity of those neurons that are sufficient for ghrelin's actions in coordinating the response to stress (GHSR neurons that coexpress $\mathrm{TH}$ or, rather, are catecholaminergic), the selectivity afforded by the TH-Cre mouse did not allow us to further narrow down the site of direct ghrelin action to one particular brain nucleus. Nonetheless, we predict that GHSR-TH-coexpressing dopaminergic neurons in the VTA are very important in ghrelin's actions in coordinating the response to stress. As mentioned, VTA dopaminergic neurons have long been known to play key roles in reward behavior of various types and mood regulation $(38,39)$, GHSRs are highly expressed in VTA dopaminergic neurons $(36,37)$, and ghrelin is known to act on these VTA neurons to influence food intake and food reward. For instance, ghrelin induces dopamine release in the NAC and increases action potential frequency in VTA dopamine neurons $(36,40,41)$. Direct ghrelin microinjection into the VTA increases intake of freely available food, intake of a rewarding diet (peanut butter) over regular chow, and operant lever pressing for a sucrose reward (20,36, 42, 43). Conversely, direct VTA microinjection of a GHSR antagonist decreases food intake in response to i.p. ghrelin and also decreases operant lever pressing for a sucrose reward normally induced by an overnight fast $(36,43)$. Also, rats containing VTA lesions consume less peanut butter but equal regular chow amounts compared with that of sham-lesioned animals and spend less time exploring tubes containing peanut butter in response to i.c.v. ghrelin (20). Additionally, selective knockdown of GHSR expression in transgenic rats expressing an antisense GHSR transcript under the control of the TH promoter decreases food intake (44).

As mentioned, besides the VTA, there are other sites of presumed usual GHSR-TH coexpression, in which TH-Cre-driven GHSR expression was observed within GHSR-null/TH mice and in which ghrelin may act in the setting of stress on mood and/or eating. Most of these sites are dopaminergic, with the possible exception of scattered cells within the nucleus of the solitary tract (which, in the rat, have been shown to include neurons with adrenergic, noradrenergic, or dopaminergic signatures; ref. 49). Direct ghrelin action on GHSR-containing dopaminergic neurons within the substantia nigra also could be mediating some of the complex food-reward behaviors we observed in the CSDSexposed mice, as neuronal projections from the substantia nigra to the dorsal striatum are involved in establishing efficient behavioral habits aimed at obtaining rewards (54). It would be even more speculative to propose a role for direct ghrelin action on dopaminergic (tuberoinfundibular) neurons within the ARC, since their known role has mainly been limited to regulatory effects on prolactin secretion (55). 
It is also intriguing to speculate whether the mechanisms herein described for stress-associated food-reward behaviors may be generalized to other reward behaviors known to occur in the setting of psychosocial stress. An association between stress and an increased risk of abusing addictive substances already is well established (56-60). For example, chronic stress has been shown to be a strong predictor of addiction vulnerability (58), and posttraumatic stress disorder is closely associated with an increased incidence of nicotine, alcohol, and other drug abuse (60). Exposure to stress has also been shown to reinstate drug-seeking behaviors in animal models and to increase chances of relapse in addictive individuals (61-63). Importantly, besides food-reward behaviors, ghrelin-signaling pathways also mediate alcohol-reward $(64,65)$, cocaine-reward (66-69), and nicotine-reward behaviors (70). Thus, ghrelin signaling, for instance, via catecholaminergic neurons, as described here, also may serve as an important link between chronic stress and drug reward.

We suspect that during evolution a link between stress, ghrelin, and eating behaviors may have instilled an important survival advantage. However, in today's modern society, activation of these ghrelin-engaged catecholamine neurons instead may contribute substantively to the altered, complex eating behaviors and development of overweight and obesity in humans exposed to chronic psychosocial stress and in humans with major depressive disorder. Future experiments should be aimed at determining the sufficiency and requirement specifically of the VTA in these actions of ghrelin as well as further clarifying the role for glucocorticoids in ghrelin action. Future studies also should focus on determining the significance of GHSR expression in TH-containing neurons at other sites, for instance, the substantia nigra and the ARC. Furthermore, our described CSDS-CPP for HFD mouse model should prove extremely useful in future studies aimed at investigating the involvement of other hormones and neuronal signaling pathways in stress-induced eating of comfort foods and other stress-related, complex eating behaviors as well as future studies aimed at developing pharmaceutical agents to curb these potentially detrimental behaviors.

\section{Methods}

Animal and housing. All procedures were performed in accordance with and with the approval of The University of Texas Southwestern Medical Center Institutional Animal Care and Use Committee guidelines, using male mice which were housed in a 12-hour-light/dark cycle, with regular chow (2016 Teklad Global 16\% Protein Rodent Diet, which provides $3.0 \mathrm{kcal} / \mathrm{g}$ [ $4.0 \mathrm{~g} \% \mathrm{fat}$ ]) and water available ad libitum, unless otherwise stated. GHSRnull mice, with their respective wild-type littermates, and GHSR-null/TH mice (with 2 GHSR-null alleles and 1 copy of TH-Cre), with their respective GHSR-null (with 2 GHSR-null alleles and no TH-Cre) and wild-type (with 2 wild-type GHSR alleles and 1 or no copies of TH-Cre) littermates, were generated as described previously $(45,46)$. All mice were on a pure C57BL/6J genetic background.

CSDS and social interaction task. CSDS is a 10-day protocol, based upon the resident-intruder paradigm, in which a test mouse is introduced into the home cage of a different aggressive CD1 mouse for a 5-minute physical encounter each day, followed by a sensory encounter for the remainder of each day, using a method reported recently (26). Control (non-CSDSexposed) mice received 5 minutes of gentle handling each day, instead of the physical exposure to an aggressive mouse experienced by the test mice, followed by a sensory encounter for the remainder of each day with a nonaggressive $\mathrm{C} 57 \mathrm{BL} / 6 \mathrm{~J}$ mouse. The social interaction task was used on day 11 and involves placing mice in an arena with a small animal cage at one end, with their movement tracked for 2.5 minutes in the absence of another mouse, followed by 2.5 minutes in the presence of a caged, unfamiliar target CD1 mouse (26). Time spent in the interaction zone and in the corners of the arena was obtained using Ethovision 3.0 software (Noldus). CSDS and the social interaction task were performed using reported methods (26).

CPP. The general CPP for HFD protocol was performed as recently reported (21). Briefly, mice underwent a 12-day conditioning protocol, in which on odd days (days $1,3,5,7,9$, and 11 of the CPP protocol) they were restricted for 20 minutes to one chamber of the CPP apparatus with HFD, and on even days (days 2, 4, 6, 8, 10, and 12 of the CPP protocol) they were restricted for 20 minutes to the other chamber of the CPP apparatus with an equal calorie amount $(2.4 \mathrm{kcal})$ of regular chow. The regular chow pellets are described above. The HFD pellets, which provide $5.56 \mathrm{kcal} / \mathrm{g}(35.8$ $\mathrm{g} \%$ of fat and $58 \mathrm{kcal} \%$ fat), were from Research Diets (Rodent Chow product no. D12331). The rest of the day was spent in their home cage with ad libitum access to regular chow. After the 12-day conditioning period, the mice were given free access for 30 minutes to the 2 chambers, each of which has a distinct wall pattern and floor texture, in the absence of either diet. Their CPP was determined by subtracting time spent in chamber paired with regular chow from time spent in chamber paired with HFD (test CPP score) and comparing the result to their preexisting place preference determined prior to conditioning (pretest CPP score). Importantly, 1 day prior to the pretest session, mice were exposed in their home cage to $1.0 \mathrm{~g} \mathrm{HFD}$ to avoid neophobia during the task.

Here, CPP was performed by administering mouse acyl-ghrelin (Pi Proteomics) ( $2 \mathrm{mg} / \mathrm{kg}$ body weight) s.c. 20 minutes before each conditioning session. Control animals instead received an equivalent volume of saline s.c. 20 minutes prior to each conditioning session. Alternatively, to assess CPP after CSDS, CSDS-exposed and control mice were placed in pretest, conditioning, and test sessions on days 13, 14-25, and 26, respectively, after the start of the 10-day CSDS procedure.

Blood glucose and hormone assessment. Blood glucose was measured at 9:00 AM from tail blood of ad libitum-fed or 24-hour-fasted mice using a OneTouch Ultra glucometer (LifeScan). Ghrelin levels were assessed at 11:00 AM, 1 day after the social interaction task using tail blood processed by the addition of EDTA, PHMB, and $\mathrm{HCl}$, as previously described (21). Corticosterone levels were assessed at 11:00 AM, 1 day after the social isolation task, using a distinct animal cohort, by ELISA (Assay Designs).

Brain punch sample collection and $q R T-P C R$ analysis. Mice were euthanized by live decapitation. Brains were extracted, placed briefly in cold diethylpyrocarbonate-PBS (DEPC-PBS), and then sectioned into 1-mm coronal slices by use of a stainless steel mouse brain matrix. The coronal slices were arranged in rostral-caudal order in DEPC-PBS, and small punches of tissue corresponding to the known locations of the ARC, VTA, DG, and NAC were excised using a 15 -g needle. These brain regions were identified by comparing the coronal slices to a mouse brain atlas. Total RNA was isolated from these punches using RNA STAT-60 (Tel-Test Inc.). RNA concentrations were determined by absorbance at $260 \mathrm{~nm}$ with a Thermo Scientific Nanodrop 100 Spectrophotometer. The total RNA was treated with RNase-free DNase (Roche) and reverse-transcribed into cDNA with SuperScript II reagents (Invitrogen). Quantitative PCR was performed using an Applied Biosystems 7900HT Sequence Detection System and SYBR Green chemistry (Applied Biosystems). GHSR-specific primers were designed using Primer Express Software (PerkinElmer Life Sciences) and validated by analysis of template titration and dissociation curves. Primers mGHSR-QF1, 5'-ACCGTGATGGTATGGGTGTCG-3', and mGHSR-QR1, 5'-CACAGTGAGGCAGAAGACCG-3', amplified a product within exon 2 of the Ghsr gene. These results (which appear in Figure 3) were confirmed by a second set of mouse GHSR-specific primers, one of which is located in exon 1 of the Ghsr gene (mGHSR-QF3 [5'-ATCTCCAGTGCCAG- 
GCACTGCT-3']) and the other of which is located in exon 2 (mGHSR-GR3 [5'-AATGGGCGCGAGCAGCAGGAA-3']) of the Ghsr gene. The mRNA levels are expressed relative to the housekeeping gene $36 \mathrm{~B} 4$ and were calculated by the comparative threshold cycle $(\Delta \Delta \mathrm{Ct})$ method (71), and the data are presented as a percentage of levels observed in wild-type ARC punches.

In situ bybridization histochemistry. To prepare tissues for histological examination, mice were deeply anesthetized with i.p. injection of chloral hydrate $(500 \mathrm{mg} / \mathrm{kg})$ and subsequently perfused transcardially with DEPC-treated $0.9 \%$ PBS, followed by $10 \%$ neutral-buffered formalin. Brains were removed, stored in the same fixative for 4 to 6 hours at $4^{\circ} \mathrm{C}$, immersed in $20 \%$ sucrose in DEPC-treated PBS, $\mathrm{pH} 7.0$, at $4^{\circ} \mathrm{C}$ overnight, and cut on a freezing microtome coronally into 5 equal series at $25 \mu \mathrm{m}$. The sections were mounted on SuperFrost slides (Fisher Scientific), air dried, and stored at $-20^{\circ} \mathrm{C}$ until further processing. In situ hybridization histochemistry for Ghsr mRNA was performed as reported previously (37) using a ${ }^{33} \mathrm{P}$-labeled mouse GHSR riboprobe generated from a 916-bp fragment of cDNA amplified with GHSRspecific primers (mGHSR1047, 5'-GTGGTGTTTGCTTTCATCCTC-3', and mGHSR1962, 5'-CATGCTCAAATTAAATGCATCC-3'). In situ hybridization histochemistry patterns were visualized first on autoradiographic film and then by observing slides dipped in photographic emulsion for direct, cellular visualization. Histological detail was noted by examining adjacent brain sections stained for thionin. Brain sections were viewed with both a Zeiss Axioskop and a Zeiss Stemi 2000-C dissecting microscope using both bright-field and dark-field optics. Photomicrographs were produced with a Zeiss digital camera attached to the microscopes and a Dell desktop computer. An image editing software program, Adobe PhotoShop 7.0, was used to combine the photomicrographs into plates, adjust contrast and brightness, and remove any obvious dust from the dark-field images. Photomicrographs taken from representative wild-type, GHSR-null, and GHSRnull/TH mice at the levels of the midbrain, ARC, and SCN are included in Supplemental Figure 1 and demonstrate reexpression of Ghsr mRNA within a subset of VTA and ARC neurons from GHSR-null/TH mice. Reexpression in the VTA of GHSR-null/TH mice had also been shown previously using a different mouse GHSR-specific riboprobe (47). The mouse GHSR-specific riboprobe also detected TH-Cre-driven GHSR expression in the substantia nigra, a few other hypothalamic nuclei (anteroventral periventricular nucleus, dorsomedial nucleus, and capsule of the ventromedial nucleus), and the scattered nucleus of the solitary tract (caudal brainstem cells) from GHSRnull/TH mice (data not shown).

Ghrelin-induced food intake. Mice were injected with saline or ghrelin $2 \mathrm{mg} / \mathrm{kg}$ body weight s.c. at 11:00 AM and intake of regular chow over the next 2 hours was measured.

Statistics. Two-way ANOVA was performed when analyzing the effects of genotype and treatment on social interaction scores, plasma ghrelin and corticosterone levels, food intake and body weight changes during CPP, ghrelin-induced acute food intake, and blood glucose levels. Significant effects of treatment (CSDS vs. control non-CSDS, ghrelin vs. saline, or ad libitum-fed vs. 24-hour fast) and significant genotype $\mathrm{X}$ treatment interactions are indicated in the appropriate figure panels. If significant genotype $\mathrm{X}$ treatment interactions were found, a secondary statistical analysis was performed using 1-way ANOVA. Statistical differences among groups resulting from this secondary analysis are indicated in the appropriate figure legends and panels. Alternatively, if no significant interaction between genotype and treatment was observed, statistical effects of one or both factors individually were determined using 2 -tailed $t$ tests, and statistically significant differences were denoted. Tukey-Kramer post-hoc analysis was used for all comparisons with significant $P$ values.

One-way ANOVA was used to analyze the effect of genotype (wild-type vs. GHSR-null vs. GHSR-null/TH) on social interaction scores. The 2-tailed, paired $t$ test was used to compare pretest versus test CPP scores within each CPP group.

Data are expressed as mean \pm SEM. $P<0.05$ was considered statistically significant, whereas $P>0.05$ was considered not significant. Analyses were performed using GraphPad Prism 5.0.

\section{Acknowledgments}

We acknowledge Chelsea Clemens, Siegfried Meier, Charlotte E. Lee, and Sherry Rovinsky for technical assistance. These studies were made possible through the support of NIH grants 1R01DA024680, 1R01MH085298, 1K08DK068069, and 1K08MH084058; The University of Texas Southwestern Medical Center Disease-Oriented Clinical Scholars Program; grants from the Klarman Family Foundation and the Foundation for Prader Willi Research; and a Young Investigator Award from NARSAD. We also thank The University of Texas Southwestern Medical Center Mouse Behavioral Core (NIH grants PL1 DK081182 and UL1RR024923).

Received for publication February 18, 2011, and accepted in revised form April 13, 2011.

Address correspondence to: Jeffrey M. Zigman, UT Southwestern Medical Center, 5323 Harry Hines Blvd., Dallas, Texas 75390-9077, USA. Phone: 214.648.8621; Fax: 214.648.5612; E-mail: jeffrey. zigman@utsouthwestern.edu.

Mario Perello's present address is: Laboratory of Neurophysiology, Multidisciplinary Institute of Cell Biology (IMBICE-CONICET/ CICPBA), La Plata, Buenos Aires, Argentina.
1. Oliver G, Wardle J. Perceived effects of stress on food choice. Physiol Behav. 1999;66(3):511-515.

2. Gibson EL. Emotional influences on food choice: sensory, physiological and psychological pathways. Physiol Behav. 2006;89(1):53-61.

3. Dallman MF. Stress-induced obesity and the emotional nervous system. Trends Endocrinol Metab. 2010;21(3):159-165.

4. American Psychiatric Association. Diagnostic and Statistical Manual of Mental Disorders. 4th Ed., Text Revision. Washington, DC, USA: American Psychiatric Association; 2000.

5. Novick JS, et al. Clinical and demographic features of atypical depression in outpatients with major depressive disorder: preliminary findings from STAR*D.J Clin Psychiatry. 2005;66(8):1002-1011.

6. Richardson LP, et al. A longitudinal evaluation of adolescent depression and adult obesity. Arch Pediatr Adolesc Med. 2003;157(8):739-745.

7. Vieweg WV, et al. Posttraumatic stress disorder and body mass index in military veterans. Preliminary findings. Prog Neuropsychopharmacol Biol Psychiatry. 2006;30(6):1150-1154.

8. Pecoraro N, Reyes F, Gomez F, Bhargava A, Dallman MF. Chronic stress promotes palatable feeding, which reduces signs of stress: feedforward and feedback effects of chronic stress. Endocrinology. 2004;145(8):3754-3762.

9. Melhorn SJ, et al. Meal patterns and hypothalamic NPY expression during chronic social stress and recovery. Am J Physiol Regul Integr Comp Physiol. 2010; 299(3):R813-R822.

10. Pankevich DE, Teegarden SL, Hedin AD, Jensen CL, Bale TL. Caloric restriction experience reprograms stress and orexigenic pathways and promotes binge eating. J Neurosci. 2010;30(48):16399-16407.

11. Teegarden SL, Bale TL. Effects of stress on dietary preference and intake are dependent on access and stress sensitivity. Physiol Behav. 2008; 93(4-5):713-723.
12. Kojima M, Hosoda H, Date Y, Nakazato M, Matsuo H, Kangawa K. Ghrelin is a growth-hormonereleasing acylated peptide from stomach. Nature. 1999;402(6762):656-660.

13. Sakata I, et al. Characterization of a novel ghrelin cell reporter mouse. Regul Pept. 2009;155(1-3):91-98.

14. Sakata I, et al. Ghrelin-producing cells exist as two types of cells, closed- and opened-type cells, in the rat gastrointestinal tract. Peptides. 2002;23(3):531-536.

15. Dornonville de la Cour C, et al. A-like cells in the rat stomach contain ghrelin and do not operate under gastrin control. Regul Pept. 2001;99(2-3):141-150.

16. Nakazato $M$, et al. A role for ghrelin in the central regulation of feeding. Nature. 2001;409(6817):194-198.

17. Tschop M, Smiley DL, Heiman ML. Ghrelin induces adiposity in rodents. Nature. 2000; 407(6806):908-913

18. Wren AM, et al. Ghrelin enhances appetite and increases food intake in humans. J Clin Endocrinol Metab. 2001;86(12):5992. 
19. Disse E, et al. Peripheral ghrelin enhances sweet taste food consumption and preference, regardless of its caloric content. Physiol Behav. 2010;101(2):277-281.

20. Egecioglu E, et al. Ghrelin increases intake of rewarding food in rodents. Addict Biol. 2010;15(3):304-311.

21. Perello $M$, et al. Ghrelin increases the rewarding value of high-fat diet in an orexin-dependent manner. Biol Psychiatry. 2010;67(9):880-886.

22. Shimbara T, et al. Central administration of ghrelin preferentially enhances fat ingestion. Neurosci Lett. 2004;369(1):75-79.

23. Disse E, et al. Systemic ghrelin and reward: effect of cholinergic blockade. Physiol Behav. 2011; 102(5):481-484.

24. Skibicka KP, Hansson C, Egecioglu E, Dickson SL. Role of ghrelin in food reward: impact of ghrelin on sucrose self-administration and mesolimbic dopamine and acetylcholine receptor gene expression [published online ahead of print February 11, 2011]. Addict Biol. doi:10.1111/j.1369-1600.2010.00294.x.

25. Malik S, McGlone F, Bedrossian D, Dagher A. Ghrelin modulates brain activity in areas that control appetitive behavior. Cell Metab. 2008;7(5):400-409.

26. Lutter $M$, et al. The orexigenic hormone ghrelin defends against depressive symptoms of chronic stress. Nat Neurosci. 2008;11(7):752-753.

27. Asakawa A, et al. A role of ghrelin in neuroendocrine and behavioral responses to stress in mice. Neuroendocrinology. 2001;74(3):143-147.

28. Kristenssson E, et al. Acute psychological stress raises plasma ghrelin in the rat. Regul Pept. 2006; 134(2-3):114-117.

29. Ochi M, et al. Effect of chronic stress on gastric emptying and plasma ghrelin levels in rats. Life Sci. 2008;82(15-16):862-868.

30. Rouach V, et al. The acute ghrelin response to a psychological stress challenge does not predict the poststress urge to eat. Psychoneuroendocrinology. 2007; 32(6):693-702.

31. Chuang JC, Zigman JM. Ghrelin's roles in stress, mood, and anxiety regulation. Int J Pept. 2010; 2010(pii):460549.

32. Berton O, et al. Essential role of BDNF in the mesolimbic dopamine pathway in social defeat stress. Science. 2006;311(5762):864-868.

33. Nestler EJ, Hyman SE. Animal models of neuropsychiatric disorders. Nat Neurosci. 2010;13(10):1161-1169.

34. Chuang JC, et al. Chronic social defeat stress disrupts regulation of lipid synthesis. J Lipid Res. 2010;51(6):1344-1353

35. Chuang JC, et al. A beta3-adrenergic-leptin-melanocortin circuit regulates behavioral and metabolic changes induced by chronic stress. Biol Psychiatry. 2010;67(11):1075-1082.

36. Abizaid A, et al. Ghrelin modulates the activity and synaptic input organization of midbrain dopamine neurons while promoting appetite. J Clin Invest. 2006;116(12):3229-3239.

37. Zigman JM, Jones JE, Lee CE, Saper CB, Elmquist JK. Expression of ghrelin receptor mRNA in the rat and the mouse brain. J Comp Neurol. 2006; 494(3):528-548.

38. NestlerEJ, Carlezon WAJr. The mesolimbic dopamine reward circuit in depression. Biol Psychiatry. 2006; 59(12):1151-1159.

39. Krishnan V, et al. Molecular adaptations underlying susceptibility and resistance to social defeat in brain reward regions. Cell. 2007;131(2):391-404.

40. Jerlhag E, Egecioglu E, Dickson SL, Douhan A, Svensson L, Engel JA. Ghrelin administration into tegmental areas stimulates locomotor activity and increases extracellular concentration of dopamine in the nucleus accumbens. Addict Biol. 2007;12(1):6-16.

41. Jerlhag E, Egecioglu E, Dickson SL, Andersson M, Svensson L, Engel JA. Ghrelin stimulates locomotor activity and accumbal dopamine-overflow via central cholinergic systems in mice: implications for its involvement in brain reward. Addict Biol. 2006; 11(1):45-54.

42. Naleid AM, Grace MK, Cummings DE, Levine AS. Ghrelin induces feeding in the mesolimbic reward pathway between the ventral tegmental area and the nucleus accumbens. Peptides. 2005; 26(11):2274-2279.

43. Skibicka KP, Hansson C, Alvarez-Crespo M, Friberg PA, Dickson SL. Ghrelin directly targets the ventral tegmental area to increase food motivation. Neuroscience. 2011;180:129-137.

44. Shuto Y, et al. Hypothalamic growth hormone secretagogue receptor regulates growth hormone secretion, feeding, and adiposity. J Clin Invest. 2002;109(11):1429-1436

45. Zigman JM, et al. Mice lacking ghrelin receptors resist the development of diet-induced obesity. J Clin Invest. 2005;115(12):3564-3572.

46. Savitt JM, Jang SS, Mu W, Dawson VL, Dawson TM. Bcl-x is required for proper development of the mouse substantia nigra. J Neurosci. 2005;25(29):6721-6728.

47. Andrews ZB, et al. Ghrelin promotes and protects nigrostriatal dopamine function via a UCP2dependent mitochondrial mechanism. J Neurosci. 2009;29(45):14057-14065.

48. Willesen MG, Kristensen P, Romer J. Co-localization of growth hormone secretagogue receptor and NPY mRNA in the arcuate nucleus of the rat. Neuroendocrinology. 1999;70(5):306-316

49. Hokfelt T, Martensson R, Bjorklund A, Kleinau $\mathrm{S}$, Goldstein M. Distributional maps of tyrosinehydroxylase-immunoreactive neurons in the rat brain. In: Bjorklund A, Hokfelt T, eds. Handbook of Chemical Neuroanatomy. Vol 2: Classical Transmitters in the CNS, Part I. Amsterdam, The Netherlands: Elsevier Science Publishers B.V.; 1984:277-379.

50. Longo KA, et al. Improved insulin sensitivity and metabolic flexibility in ghrelin receptor knockout mice. Regul Pept. 2008;150(1-3):55-61.

51. Zhao TJ, et al. Ghrelin secretion stimulated by \{beta\}1-adrenergic receptors in cultured ghrelinoma cells and in fasted mice. Proc Natl Acad Sci US A 2010;107(36):15868-15873

52. Mundinger TO, Cummings DE, Taborsky GJ Jr Direct stimulation of ghrelin secretion by sympathetic nerves. Endocrinology. 2006;147(6):2893-2901.

53. Sgoifo A, et al. Social stress, autonomic neural activation, and cardiac activity in rats. Neurosci Biobehav Rev. 1999;23(7):915-923.

54. Barnes TD, Kubota Y, Hu D, Jin DZ, Graybiel AM. Activity of striatal neurons reflects dynamic encoding and recoding of procedural memories. Nature. 2005;437(7062):1158-1161.

55. Mohankumar SM, et al. Chronic estradiol exposure induces oxidative stress in the hypothalamus to decrease hypothalamic dopamine and cause hyperprolactinemia. Am J Physiol Regul Integr Comp Physiol. 2011;300(3):R693-R699.

56. Cooper ML, Russell M, Frone MR. Work stress and alcohol effects: a test of stress-induced drinking. J Health Soc Behav. 1990;31(3):260-276

57. Kandel DB, et al. Psychiatric disorders associated with substance use among children and adolescents: findings from the Methods for the Epidemiology of Child and Adolescent Mental Disorders (MECA) Study. J Abnorm Child Psychol. 1997;25(2):121-132.

58. King CA, Ghaziuddin N, McGovern L, Brand E, Hill E, Naylor M. Predictors of comorbid alcohol and substance abuse in depressed adolescents. J Am Acad Child Adolesc Psychiatry. 1996;35(6):743-751.

59. Reed PL, Anthony JC, Breslau N. Incidence of drug problems in young adults exposed to trauma and posttraumatic stress disorder: do early life experiences and predispositions matter? Arch Gen Psychiatry. 2007;64(12):1435-1442.

60. Breslau N, Davis GC, Schultz LR. Posttraumatic stress disorder and the incidence of nicotine, alcohol, and other drug disorders in persons who have experienced trauma. Arch Gen Psychiatry. 2003; 60(3):289-294.

61 . Sinha R. The role of stress in addiction relapse. Curr Psychiatry Rep. 2007;9(5):388-395.

62. Shaham Y, Shalev U, Lu L, De Wit H, Stewart J. The reinstatement model of drug relapse: history, methodology and major findings. Psychopharmacology (Berl). 2003;168(1-2):3-20.

63. Sanchez CJ, Sorg BA. Conditioned fear stimuli reinstate cocaine-induced conditioned place preference. Brain Res. 2001;908(1):86-92.

64. Addolorato G, et al. Relationship between ghrelin levels, alcohol craving, and nutritional status in current alcoholic patients. Alcohol Clin Exp Res. 2006; 30(11):1933-1937.

65. Jerlhag E, et al. Requirement of central ghrelin signaling for alcohol reward. Proc Natl Acad Sci U S A. 2009;106(27):11318-11323.

66. Jerlhag E, Egecioglu E, Dickson SL, Engel JA. Ghrelin receptor antagonism attenuates cocaine- and amphetamine-induced locomotor stimulation, accumbal dopamine release, and conditioned place preference. Psychopharmacology (Berl). 2010;211(4):415-422.

67 . Tessari $\mathrm{M}$, et al. Correlation between serum ghrelin levels and cocaine-seeking behaviour triggered by cocaine-associated conditioned stimuli in rats. Addict Biol. 2007;12(1):22-29.

68. Davis KW, Wellman PJ, Clifford PS. Augmented cocaine conditioned place preference in rats pretreated with systemic ghrelin. Regul Pept. 2007; 140(3):148-152.

69. Wellman PJ, Davis KW, Nation JR. Augmentation of cocaine hyperactivity in rats by systemic ghrelin. Regul Pept. 2005;125(1-3):151-154.

70. Jerlhag E, Engel JA. Ghrelin receptor antagonism attenuates nicotine-induced locomotor stimulation, accumbal dopamine release and conditioned place preference in mice [published online ahead of print February 8, 2011]. Drug Alcohol Depend. doi:10.1016/j.drugalcdep.2011.01.010.

71. Kurrasch DM, Huang J, Wilkie TM, Repa JJ. Quantitative real-time polymerase chain reaction measurement of regulators of G-protein signaling mRNA levels in mouse tissues. Methods Enzymol. 2004; 389:3-15. 